\title{
Clinical observation of tongxinluo capsules in the treatment of diabetic peripheral neuropathy.
}

\author{
Qian Xiao" $^{1 \#}$, Fang Liu ${ }^{2 \#}$, Qian Zhao ${ }^{1}$, Feng Chen ${ }^{1}$, Yong Zhou ${ }^{*}$ \\ ${ }^{1}$ Department of Endocrinology, Xuhui District Dahua Hospital, Shanghai, PR China \\ ${ }^{2}$ Department of Endocrinology, Shanghai Jiaotong University Affiliated Sixth People's Hospital, Shanghai, PR China \\ \#These authors contributed equally to this paper
}

\begin{abstract}
Objective: To observe the clinical effect of tongxinluo capsules in the treatment of diabetic peripheral neuropathy.

Methods: A total of 132 patients with diabetic peripheral neuropathy in our hospital from June 2014 to June 2017 were selected as subjects. Using a random even-odd number method, the patients were divided into control $(n=66)$ and treatment $(n=66)$ groups. The patients in the control group were treated with mecobalamin, whereas the patients in the treatment group were treated with tongxinluo capsules. Clinical efficacy, blood glucose level change, electromyography nerve conduction velocity, and clinical signs of improvement were compared before and after treatment.

Results: The total effective rate was significantly higher in the treatment group than in the control group $(\mathbf{P}<0.05)$. Meanwhile, the fasting blood glucose, postprandial $2 \mathrm{~h}$ blood glucose, and glycosylated hemoglobin levels were significantly lower in the treatment group than in the control group $(P<0.05)$. Moreover, motor and sensory nerve conduction velocities were significantly higher in the treatment group than in the control group $(\mathbf{P}<\mathbf{0 . 0 5})$. The total effective rate of clinical symptom improvement was significantly lower in the treatment group than in the control group $(\mathbf{P}<0.05)$.

Conclusion: Tongxinluo capsules can alleviate clinical symptoms, as well as improve therapeutic effects, blood glucose levels, and nerve conduction velocity, in patients with diabetic peripheral neuropathy. The capsules can be used as an ideal drug and merits popularization.
\end{abstract}

Keywords: Tongxinluo capsules, Diabetic peripheral neuropathy, Clinical efficacy. Accepted on November 7, 2017

\section{Introduction}

Diabetes is a highly common clinical disease. It is a metabolic disease caused by biological damage, insulin secretion defect, or both. The significant characteristic of patients with diabetes is hyperglycemia [1]. The incidence of Diabetic Peripheral Neuropathy (DPN) has increased with time. In the past, the patients with DPN were treated with aldose reductase inhibitors, vasodilators, vitamins, and other drugs [2,3]. However, the efficacy of these drugs needs further exploration and confirmation. In recent years, traditional Chinese medicines and Chinese patent medicines have been widely used and achieved remarkable therapeutic effects. In our study, 132 cases of DPN admitted to our hospital were selected as research subjects. The therapeutic effect is reported as follows.

\section{Materials and Methods}

\section{General information}

A total of 132 cases of DPN admitted to our hospital from June 2014 to June 2017 were recruited to our study as research subjects. Our inclusion criteria were as follows: fulfilment of the diagnostic criteria for diabetes and DPN established by the American Diabetes Association, fulfilment of the diagnostic criteria for deficiency of Yang collaterals, knowledge of the study, and informed consent.

Our exclusion criteria were as follows: 30-70 y of age; peripheral neuropathy caused by other diseases; concomitant myocardial infarction, cerebral infarction, malignant tumors, or mental disorders; severe infections and acute complication within 30 days; pregnant or lactating; severe liver and kidney dysfunctions; digestion ulcers; severe diarrhea; and other related diseases. 
Using the random even-odd number method, the patients were divided into the control group $(n=66)$ and the treatment group $(n=66)$. The control group comprised 31 males and 35 females aged 43-70 y (average: $57.5 \pm 6.2$ y) with disease courses lasting for 1-7 y (average: $3.2 \pm 1 \mathrm{y}$ ). Meanwhile, the treatment group was composed of 30 males and 36 females aged 44-72 y (average: $59.3 \pm 6.1 \mathrm{y}$ ) with disease courses lasting for 1-8 y (average: $3.4 \pm 1.2 \mathrm{y}$ ). The natural data were compared between the two groups, and the difference was not significant $(\mathrm{P}>0.05)$.

\section{Method}

Patients' daily diets were adjusted and controlled. They followed the doctor's advice and took insulin and sulfonylureas. After the blood glucose levels were thoroughly controlled, comparative treatment was performed. The patients were not given analgesic and vasodilators during treatment. The patients in the control group were treated with mecobalamin (SFDA approval number H20070279, Shandong Renhe Pharmaceutical Co. Ltd.; specifications: chemical medicine, $0.5 \mathrm{mg}$ ) at $500 \mu \mathrm{g} / \mathrm{dose}$, thrice daily, for 2 months. By contrast, the patients in the treatment group were treated with Tongxinluo capsules (SFDA approval number Z19980015, Shijiazhuang Yiling pharmaceutical Limited by Share Ltd.; specifications: Chinese medicine, $0.26 \mathrm{~g} /$ capsule) at a dosage of 4 capsules, thrice daily, for 2 months.

\section{Observation index}

Venous blood was drawn from the patients in the two groups 2 months before and after treatment. Fasting blood glucose and postprandial $2 \mathrm{~h}$ blood glucose levels were detected using the glucose oxidase method. Glycated hemoglobin levels were detected using the microcolumn method. Sensory and motor nerve conduction velocities were determined using the KeypointV3.00 myoelectricity/evoked potential instrument. Improvement in clinical symptoms and therapeutic effect was evaluated.

\section{Efficacy evaluation criteria}

Evaluation criteria for the clinical signs and symptoms were as follows: On the basis of the "new Chinese medicine clinical research guiding principles," clinical symptom improvement was evaluated considering the clinical signs of Achilles tendon reflex and tactile reflex before and after treatment as the bias. The evaluation was graded as follows: "healed" (main clinical symptoms and signs completely or almost disappeared after treatment with symptom score decreased by $>95 \%$ ), "excellent" (clinical symptoms and signs improved significantly after treatment with symptom score decreased by $>75 \%$ ), "effective" (clinical symptoms alleviated after treatment with symptom score decreased by $>30 \%$ ), and "invalid" (clinical symptoms not obviously altered or continuously aggravated after treatment with symptom score decreased by $<30 \%$ ). The total effective rate was determine as the sum of the percentages for "healed," "excellent," and "effective."

Evaluation criteria for the therapeutic effect were as follows: "excellent" (all clinical symptoms disappeared, tendon reflex returned to normal, and the nerve conduction velocity increased by $\geq 5 \mathrm{~m} / \mathrm{s}$ after treatment), "effective" (clinical symptoms improved, tendon reflex returned to near normal, and nerve conduction velocity increased by $<5 \mathrm{~m} / \mathrm{s}$ after treatment), and "invalid" (clinical symptoms, tendon reflex, and nerve conduction velocity did not change or were aggravated after treatment). The total effective rate was calculated using the formula Total effective rate $=($ excellent + effective)/total number $\times 100 \%$.

\section{Statistical processing}

Data were processed using SPSS 22.0. Measurement data (including glucose metabolic index and sensory nerve conduction velocity) were compared by $\overline{\mathrm{x}} \pm \mathrm{S}$ and tested using the t-test. Count data (including clinical symptom improvement and total effective rate) were compared using percentages (\%) and evaluated with the $\chi^{2}$ test. $\mathrm{P}<0.05$ indicated that the difference between the two groups was statistically significant.

\section{Result}

\section{Glucose metabolism indexes in the two groups before and after treatment}

Fasting blood glucose, postprandial $2 \mathrm{~h}$ blood glucose, and glycosylated hemoglobin levels of the patients in the two groups were compared and showed no significant difference $(\mathrm{P}>0.05)$. After treatment, the values of the above-mentioned indexes in the treatment group were significantly higher than those in the control group $(\mathrm{P}<0.05)$ (Table 1).

Table 1. Glucose metabolism indexes in the two groups before and after treatment.

\begin{tabular}{|c|c|c|c|c|c|c|}
\hline \multirow[t]{2}{*}{ Group } & \multicolumn{2}{|c|}{$\begin{array}{l}\text { Fasting blood } \\
\text { glucose (mmol/L) }\end{array}$} & \multicolumn{2}{|c|}{$\begin{array}{l}\text { Postprandial } 2 \quad \text { h } \\
\text { blood } \\
\text { (mmol/L) }\end{array}$} & \multicolumn{2}{|c|}{$\begin{array}{l}\text { Glycosylated } \\
\text { hemoglobin (\%) }\end{array}$} \\
\hline & Before & After & Before & After & Before & After \\
\hline Control & $8.6 \pm 1.7$ & $7.5 \pm 0.9$ & $10.7 \pm 1.7$ & $8.6 \pm 1.7$ & $8.3 \pm 1.1$ & $7.2 \pm 0.9$ \\
\hline $\begin{array}{l}\text { Treatmen } \\
t\end{array}$ & $8.8 \pm 0.8$ & $6.2 \pm 0.8$ & $10.9 \pm 1.7$ & $6.7 \pm 1.5$ & $8.5 \pm 1.1$ & $6.4 \pm 1.0$ \\
\hline $\mathrm{t}$ & 0.8647 & 8.7706 & 0.6758 & 6.8083 & 1.0444 & 4.8308 \\
\hline$P$ & 0.3887 & 0.0000 & 0.5003 & 0.0000 & 0.2982 & 0.0000 \\
\hline
\end{tabular}

\section{Electromyography nerve conduction velocity in the two groups before and after treatment}

Before treatment, the motor and sensory nerve conduction velocities of the patients did not significantly differ between the treatment and control groups $(\mathrm{P}>0.05)$. After treatment, the 
results in the treatment group were significantly higher than those in the control group $(\mathrm{P}<0.05)$ (Table 2$)$.

Table 2. Electromyography nerve conduction velocity of patients in the two groups before and after treatment. Note: ${ }^{*}$ Compared with before treatment, $P<0.05$; ${ }^{\#}$ compared with the control group, $P<0.05$.

\begin{tabular}{|c|c|c|c|c|c|}
\hline \multirow[t]{2}{*}{ Group } & \multirow[t]{2}{*}{ Time } & \multicolumn{2}{|c|}{ Sensory nerve conduction velocity $(\mathrm{m} / \mathrm{s})$} & \multicolumn{2}{|c|}{ Motor nerve conduction velocity (m/s) } \\
\hline & & Tibial nerve & Common peroneal nerve & Tibial nerve & Common peroneal nerve \\
\hline \multirow[t]{2}{*}{ Control } & Before & $36.6 \pm 4.4$ & $35.3 \pm 5.1$ & $36.6 \pm 4.0$ & $36.9 \pm 3.6$ \\
\hline & After & $42.3 \pm 5.7$ & $38.3 \pm 5.3$ & $38.7 \pm 4.2$ & $40.5 \pm 3.9$ \\
\hline \multirow[t]{2}{*}{ Treatment } & Before & $37.9 \pm 5.1$ & $35.6 \pm 5.6$ & $37.3 \pm 4.2$ & $35.9 \pm 3.2$ \\
\hline & After & $48.3 \pm 6.1^{\star \#}$ & $44.6 \pm 5.4^{*} \#$ & $46.9 \pm 4.0^{* \#}$ & $47.1 \pm 4.3^{\star \#}$ \\
\hline
\end{tabular}

\section{Improvement in the major clinical symptoms and signs in the two groups}

Total effective rate of the clinical symptom and sign improvement in the treatment group $(92.4 \%)$ was significantly higher than that in the control group $(77.3 \%)(\mathrm{P}<0.05)$ (Table 3).

Table 3. Main clinical symptoms and signs in the two groups ( $(\%))$.

\begin{tabular}{llllll}
\hline Group & Cured & Excellent & Effective & Invalid & $\begin{array}{l}\text { Total } \\
\text { effective } \\
\text { rate }\end{array}$ \\
\hline $\begin{array}{l}\text { Treatment } \\
\text { group }(n=66)\end{array}$ & $28(42.4)$ & $20(30.3)$ & $13(19.7)$ & $5(7.6)$ & $61(92.4)$ \\
\hline $\begin{array}{l}\text { Control } \\
\text { group }(n=66)\end{array}$ & $24(36.4)$ & $17(25.8)$ & $10(15.2)$ & $15(22.7)$ & $51(77.3)$ \\
\hline$X^{2}$ & & & & 5.8929 \\
\hline$P$ & & & & 0.0152 \\
\hline
\end{tabular}

\section{Clinical efficacy in the two groups}

Total effective rate was significantly lower in the control group than in the treatment group $(\mathrm{P}<0.05)$ (Table 4$)$.

Table 4. Clinical efficacy in the two groups (n(\%)).

\begin{tabular}{lllll}
\hline Group & Excellent & Effective & Invalid & Total effective rate \\
\hline $\begin{array}{l}\text { Treatment } \\
\text { group }\end{array}$ & $34(51.5)$ & $28(42.4)$ & $4(6.1)$ & $62(93.9)$ \\
\hline Control group & $29(43.9)$ & $24(36.3)$ & $13(19.7)$ & $53(80.3)$ \\
\hline$X^{2}$ & & & 5.4691 \\
\hline$P$ & & & 0.0194 \\
\hline
\end{tabular}

\section{Discussion}

For patients with diabetes, high-level hyperglycemia can cause chronic damage to tissues and functions. If timely treatment and control measures are not applied, complications tend to ensue. The most common complication is DPN; however, its pathogenesis is unclear. The specific etiology involves neurotrophic disorder, metabolic disorders, and vascular damage [4]. In addition, clinical practice found that genetic factors, autoimmune damage, and oxygen free-radical damage are among the main reasons of DPN. With the development of traditional Chinese medicine in recent years, the clinical efficacy and safety of Chinese traditional medicine in treating DPN have been affirmed by the majority of patients and doctors $[5,6]$.

The tongxinluo capsule belongs under Chinese patent medicine. Its main component ginseng belongs to sovereign drug and operates by tonifying the blood [7]. Meanwhile, the other components of leech and scorpion belong under adjuvant drug and function by helping activate blood and dredge collaterals, as well as exhibit an antispasmodic activity [8]. Red peony, centipede, and periostracum cicada also belong to adjuvant drugs. These components help cool blood to dissipate stasis, relieve stasis and alleviate pain, expel wind to relieve spasm, and relieve spasm by subduing liver-wind [9]. Meanwhile, the borneol can separate freely and dredge stasis and exerts its therapeutic effect by bringing the abovementioned drugs to the collaterals. The combination of the above-mentioned drugs can help dredge collaterals and restore blood circulation. Modern pharmacology has confirmed that the tongxinluo capsule can maintain vascular endothelial function and decrease vascular spasm [10]. As a result, peripheral tissue blood supply and nerve hypoxic ischemia are improved, and nerve injury repair is accelerated.

\section{Conclusion}

Our study results revealed that the blood glucose metabolic index, electromyography nerve conduction velocity, clinical symptom and sign improvement, and overall efficacy significantly differed between the treatment and control groups $(\mathrm{P}<0.05)$. Our data sufficiently showed that tongxinluo capsules are beneficial for alleviating clinical symptoms, improving therapeutic effects, and enhancing the blood glucose levels and electromyography nerve conduction velocity in the treatment of DPN. As such, the tongxinluo capsule is an ideal drug. 


\section{References}

1. Lee K. Effects of whole-body vibration therapy on perception thresholds of type 2 diabetic patients with peripheral neuropathy: a randomized controlled trial. J Phys Ther Sci 2017; 29: 1684-1688.

2. Hafiz TA, Mubaraki MA. The potential role of Ziziphus spina-christi leaf extracts against Plasmodium bergheiinduced liver and spleen injury. Biomed Res India 2016; 27: 1027-1032.

3. Lima KCA, Borges LDS, Hatanaka E, Rolim LC, de Freitas PB. Grip force control and hand dexterity are impaired in individuals with diabetic peripheral neuropathy. Neurosci Lett 2017; 659: 54-59.

4. Lee EC, Kim MO, Roh GH, Hong SE. Effects of Exercise on Neuropathy in Streptozotocin-Induced Diabetic Rats. Ann Rehabil Med 2017; 41: 402-412.

5. Colak MC, Colak C, Erdil N, Sandal S. Potential risk factors for early large pleural effusion after coronary artery bypass grafting surgery. Biomed Res India 2017; 28: 625-629.

6. Hu G, Zhai F, Mo F, He L, Shen W, Wang H. Effectiveness and feasibility of nailfold microcirculation test to screen for diabetic peripheral neuropathy. Diabetes Res Clin Pract 2017; 131: 42-48.
7. Fang JH, Zhang SH, Yu XM, Yang Y. Effects of Quercetin and melatonin in pregnant and gestational diabetic women. Lat Am J Pharm 2016; 35: 1420-1425.

8. Li YB, Wu Q, Liu J, Fan YZ, Yu KF, Cai Y. miR 199a 3p is involved in the pathogenesis and progression of diabetic neuropathy through downregulation of SerpinE2. Mol Med Rep 2017; 16: 2417-2424.

9. Zhang J, Zhang Z, Yang B, Jing X, Zhong W, Tang H. Synergistic effect of melatonin and atorvastatin in type 1 diabetic and ischemic injury cardiomyopathic sprague dawley rats. Lat Am J Pharm 2016; 35: 1719-1724.

10. Mahdi TN, Gholamhassan V, Hossein AE, Vida H. Acute and chronic effects of opiates and dopamine on yawning, penile erection and genital grooming behaviors in male Wistar rats. Biomed Res India 2017; 28: 6931-6936.

\section{${ }^{*}$ Correspondence to}

Yong Zhou

Department of Endocrinology

Xuhui District Dahua Hospital

PR China 